\title{
Kinetics of Hydrolytic Degradation of the Nitrosyl Iron Complex with Cysteamine
}

\author{
Anatoly I. Kazakov*, Tatyana N. Rudneva, Ludmila S. Kurochkina, Valery V. Dubikhin, \\ Nataliya A. Sanina \\ Institute of Problems of Chemical Physics, Russian Academy of Sciences, Acad. Semenov av. 1, Chernogolovka, Moscow region, 142432 \\ Russian Federation \\ *Corresponding Author: akazakov@icp.ac.ru
}

Copyright $(\underset{0}{2013}$ Horizon Research Publishing All rights reserved.

\begin{abstract}
The hydrolytic degradation kinetics of a new promising anticancer agent - donor of NO, namely, water-soluble nitrosyl iron complex, $\left[\mathrm{Fe}_{2}\left(\mathrm{~S}(\mathrm{CH})_{2} \mathrm{NH}_{3}\right)_{2}(\mathrm{NO})_{4}\right] \mathrm{SO}_{4} \cdot 2.5 \mathrm{H}_{2} \mathrm{O}(1)$, has been studied by electrochemical and microcalorimetric methods in water-dimethylsulfoxide (DMSO) (1:1) solution. Henry's constant for NO dissolved in water-dimethylsulfoxide (DMSO) (1:1) solution has been measured. The temperature dependence of hydrolytic degradation rate constant of complex 1 has been determined. High enough rates of the hydrolytic degradation of these complexes at high negative entropy of activation can be explained by the low value of activation energy.
\end{abstract}

Keywords Nitrosyl Iron Complexes, Kinetics, Hydrolytic Degradation, Microcalorimetry, Amperometry, Henry's Law

\section{Introduction}

The latest numerous investigations show strong evidence of that NO is a key agent in many cellular functions, such as regulation of blood pressure, inhibition of the thrombocytes aggregation, immune cellular response, antimicrobial and antitumor activity, and others [1]. These discoveries gave rise to studying biologically active cellular intermediates, in particular, nitrosyl non-heme proteins, which are known to prolong the lifetime of the endogenous nitric oxide and keep its biological activity [2]. As it follows from EPR studies, these non-heme iron nitrosyl complexes with aliphatic thiols are paramagnetic complexes containing "Fe(NO)2" moiety and having a typical isotropic g-factor equal to $2.03[3,4]$. Recently synthetic models of the active sites of nitrosyl [2Fe-2S] ferredoxin have been isolated and characterized by X-ray method and they are of particular interest due to possible use as new NO-donor anticancer agents in monotherapy of tumors [5]. In particular, a water-soluble cationic binuclear tetranitrosyl iron complex with cysteamine $\left[\mathrm{Fe}_{2}\left(\mathrm{~S}(\mathrm{CH})_{2} \mathrm{NH} 3\right)_{2}(\mathrm{NO})_{4}\right] \mathrm{SO}_{4} \cdot 2.5 \mathrm{H}_{2} \mathrm{O}$ (1) exhibits high cytotoxic activity to tumor cells of breast cancer (SKBR-3 and MCF-7 lines) and to myelogenous leukemia cells (K562 line) as an inducer of apoptosis. It also shows a high antitumor effect on breast adenocarcinoma (Ca-755 line) for 9 days after treatment (the tumor growth inhibition in mice under study is equal to $71 \%$ and the animal life duration is equal to $66 \%$ ) and on Lewis lung epidermoid carcinoma (LLC) for 10 days after treatment (the tumor growth inhibition in mice under study is equal to $86 \%$ and the animal life duration is equal to $7 \%$ [6]). To determine reactivity and the mechanism of antitumor activity of the complex the study of its NO-donor activity and mechanisms of hydrolysis in conditions close to physiological, namely, in reactions with heme proteins was performed [7, 8].

The purpose of this work is to study the kinetics of hydrolytic degradation of complex 1 in media without targets with a high affinity to NO and nitrosyl intermediates formed during its decomposition in solution.

\section{Experimental}

\subsection{Materials}

Complex 1 was obtained according to the method described in $[6,9]$. Nitrogen oxide NO was obtained by the method [10]. DMSO was purified by the method [11]. Removal of oxygen from the distilled water and DMSO was performed by triple freezing-out and evacuating using a vacuum line and Shlenk's technology.

\subsection{Methods}

\subsubsection{Electrochemical Method}

The kinetics of accumulation of NO in solution formed during the hydrolytic degradation of 1 was found using an amiNO-2000 sensor electrode of in NO Nitric Oxide 
Measuring System (Innovative Instruments, Inc., Tampa, FL, USA) $[12,13]$ at $28^{\circ} \mathrm{C} .2 \mathrm{ml}$ of DMSO solution of complex 1 and $2 \mathrm{ml}$ of water were placed in the reaction vessel in such a way that there was no mixing of solvents. Then the sensor electrode was immersed in the mixture. The reaction vessel was closed with a stopper, and the latter was heat sealed with paraffin. The volume of the vessel was equal to $7.4 \mathrm{ml}$. The volume of the water-DMSO solution of complex 1 was equal to $4 \mathrm{ml}$. The sensor signal was recorded from the very moment of pouring the DMSO solution of complex 1 to the reaction vessel. When degassed distilled water was added to the vessel without mixing the layers of DMSO and water, a temperature jump of about 5 degrees was observed due to positive heat of mixing DMSO with water. Approximately 8.5 min was needed to return to initial temperature. During this time the accumulation of about $30 \mathrm{nmol}$ of $\mathrm{NO}$ in solution was recorded. This quantity is insignificant in comparison with the amount of NO to be formed at $100 \%$ conversion degree of the complex (about $1.1 \cdot 10^{5} \mathrm{nmol}$ ). The second temperature peak corresponding to heat of solution at 3 degrees was observed when DMSO and water layers were stirred. Concentration of NO in solution was measured for 2.2 hours with an increment of $0.2 \mathrm{~s}$. At the end of the record the amount of NO in solution was equal to $3.8 \cdot 10^{3} \mathrm{nmol}$. To calibrate the electrochemical sensor a $100 \mu \mathrm{M}$ standard aqueous solution of $\mathrm{NaNO}_{2}$ was used, which was added to the mixture containing $20 \mathrm{mg}$ of $\mathrm{KJ}$ (Aldrich), $2 \mathrm{ml}$ of $1 \mathrm{M}$ $\mathrm{H}_{2} \mathrm{SO}_{4}$ (reagent grade, Russian national standard, GOST 4204-77) and $18 \mathrm{ml}$ of water.

\subsubsection{Dynamic Microcalorimetry}

Hydrolytic degradation rates were determined from measuring the heat release rates in the process using an automated differential microcalorimeter (DAC-1-2) [14] developed and manufactured at the Institute of Problems of Chemical Physics RAS. The microcalorimeter can operate in isothermal conditions in a wide temperature range from room temperature up to $400^{\circ} \mathrm{C}$ and the heat release rate can be measured in the range from $10^{-5} \mathrm{~W}$ to $1 \mathrm{~W}$. Hydrolytic degradation kinetics was studied in the $29.4-66.1^{\circ} \mathrm{C}$ temperature range. The solutions of $\mathbf{1}$ were prepared for kinetic experiments as follows: a sample of $\mathbf{1}$ was placed in the glass tube into which the required amount of degassed DMSO was poured. Then tube contents the reaction mixture was stirred for 30-45 minutes with a magnetic stirrer until 1 completely dissolved in DMSO. The solution was placed into the calorimeter glass ampoule, and an equal volume of degassed $\mathrm{H}_{2} \mathrm{O}$ was added. The layers of an organic solution and water were not mixed. The ampoule was cooled down to liquid nitrogen temperature, evacuated and sealed. Then the ampoule was thawed, the water and organic layers were mixed, and the ampoule was placed in the calorimeter for kinetic experiments. In the described mode of preparation for experiments, heat released during mixing DMSO with water is consumed to heat the solution in the ampoule to room temperature. The time to warm up the ampoule to the temperature of the calorimeter is approximately $10-15 \mathrm{~min}$.
After warm-up the heat release rate was recorded.

\subsubsection{Measurement of the Solubility of NO in the Water-Dimethyl Sulfoxide Solution}

Henry's law constant $K_{h}$ for NO dissolved in a mixed water-DMSO solvent was determined using a thermostated Bourdon's vessel with a total volume $\left(V_{L}+V_{G}\right)$ of $56 \mathrm{ml}$, which allows pressure to be measured in the range of $0 \div 760$ $\mathrm{mm} \mathrm{Hg}$. In the experiment the Bourdon's vessel was filled with $32 \mathrm{ml} V_{L}$ of the mixed solvent and evacuated until bubbling from the liquid stopped. After the liquid-vapor equilibrium was attained, the partial equilibrium vapor pressure $p_{\text {vap }}$ at several temperatures was measured by a mercury manometer. Then the Bourdon's vessel was placed in liquid nitrogen and a certain amount of $\mathrm{NO}$ was frozen out from the vessel of the dimensional volume $V=29 \mathrm{ml}$. Initial pressure of NO $p_{\text {int }}$ in the dimensional volume $V$ was $510 \mathrm{~mm}$ Hg. Then the contents of the Bourdon's vessel was thawed and stirred with a magnetic stirrer for about a day up to attain the gas-liquid equilibrium. Then the Bourdon's vessel was placed in a thermostat and the sum of equilibrium partial pressures of $\mathrm{NO}$ and the solvent vapor $p_{e q}$ was measured at several temperatures. Henry's law constant was calculated using the formula

$$
K_{h}=R T V_{L} n_{G, N O} / V_{G} n_{L, N O} / \mathrm{atm} \cdot l \cdot \mathrm{mol}^{-1}
$$

where $n_{G, N O}$ is a number of moles of NO in the gas phase, which was calculated by the formula

$$
\text { (peq-pvap) } V_{G} / R T
$$

$n_{L, N O}$ is a number of moles of $\mathrm{NO}$ in the liquid phase, which was calculated using the formula

$$
\left[{ }_{\text {pint }} V \text { - }(\text { peq-pvap }) V_{G}\right] / R T
$$

\section{Results and Discussion}

\subsection{Dynamic Microcalorimetry}

According to X-ray analysis [9], complex $\mathbf{1}$ is a salt with the binuclear nitrosyl cation, in which the iron atoms coordinated in pairs by $\mathrm{NO}$ groups are bonded to each other through the bridging sulfur atoms (structural type " $\mu_{2}-\mathrm{S} "$ ). Solution of 1 in mixed of water-DMSO (1:1) solvent was used to study the hydrolysis process and to determine the rate constants of this process by means of the calorimetric method. The solvent was chosen using the requirements of the experiment, namely, relatively high concentration of complex $1(\sim 10 \mathrm{mM})$ obtained by dissolving 1 in DMSO (water solubility of 1 is about $2 \mathrm{mM}$ ) and the availability of water as a reagent for the hydrolysis reaction. The concentration of complex 1 in the mixed water-DMSO solvent was $7-8 \cdot \mathrm{mM}$.

The dependence of heat release rates on current heat, which is proportional to the conversion degree in the hydrolytic degradation reaction in the range of experimental 
temperatures is demonstrated in Fig. 1.

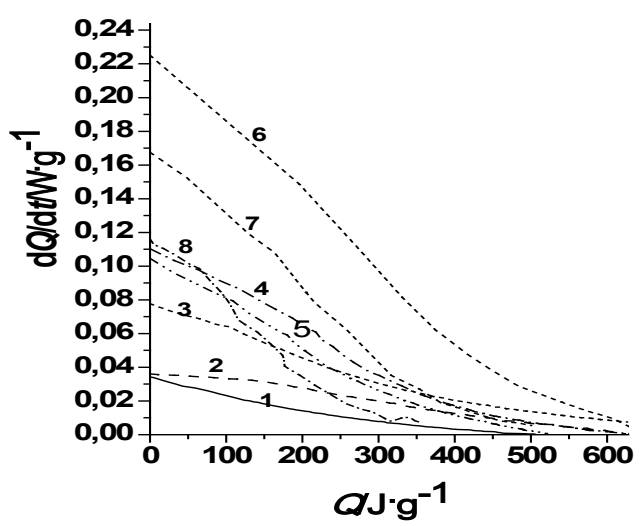

Figure 1. Heat release rate $d Q / d t$ versus current heat $Q$ in the reaction of hydrolytic degradation of the complex $1 . T\left({ }^{\circ} \mathrm{C}\right): 1-29.4 ; 2-32.2 ; 3-35.1$; $4-39.1 ; 5-42.9 ; 6-50.8 ; 7-59.2 ; 8-66.1$

It is seen that the process of hydrolytic decomposition of $\mathbf{1}$ in water-DMSO solution is described by the equation of the pseudo- first-order reaction:

$$
\mathrm{d} \eta / \mathrm{dt}=(\mathrm{dQ} / \mathrm{dt}) / \mathrm{Q} 0=\mathrm{k}(1-\eta)
$$

where $\eta=\int_{0}^{t}(\mathrm{~d} Q / \mathrm{d} t) \mathrm{d} t / \int_{0}^{\infty}(\mathrm{d} Q / \mathrm{d} t) \mathrm{d} t$ is the conversion degree; $\mathrm{d} \eta / \mathrm{d} t=(\mathrm{d} Q / \mathrm{d} t) / Q_{0}$ is the process rate, $\mathrm{d} Q / \mathrm{d} t$ is the heat release rate, $\int_{0}^{\infty}(\mathrm{d} Q / \mathrm{d} t) \mathrm{d} t$ is the total heat of hydrolytic degradation reaction.

The process of hydrolytic degradation of $\mathbf{1}$ proceeds to the considerable conversion degree with its complete destruction. Hydrolysis heat of per mole of the complex is about $290 \mathrm{~kJ}$. There is a precipitate of hydroxide iron(III) at the end of the reaction as a product of the concentration of iron ions, which is likely to be equal to the initial concentration of the complex, and the concentration of hydroxide ions to the third power is about 12 orders higher than the $\mathrm{SP}\left[\mathrm{Fe}(\mathrm{OH})_{3}\right]$. The dependence of reaction rate constant $k \mathrm{~h}^{-1}$ of hydrolytic degradation of 1 versus reciprocal temperature in the Arrhenius' equation coordinates is shown in Fig. 2.

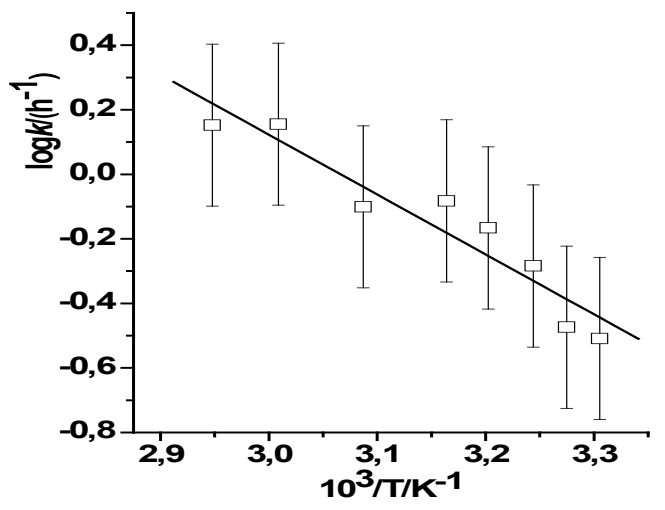

Figure 2. Dependence of logk (h-1) versus the reciprocal temperature in the reaction of hydrolytic degradation of complex 1 .
The dependence of rate constant is as follows:

$$
\left.k=10^{2.1 \pm 0.8} \exp [-(4270 \pm 570) / T)\right] / s^{-1}(r=0.95)
$$

To calculate the rate constant of the second-order reaction of $\mathbf{1}$ with water from the obtained value of $k_{I}$ it is necessary to consider that concentration of water in a solution is equal to $\sim 27.810^{3} \mathrm{~mol} \cdot \mathrm{m}^{-3}$. As a result,

$$
\left.k I I=10^{-2.3 \pm 0.8} \exp [-(4270 \pm 570) / T)\right] / m^{3} \cdot m^{-1} \cdot s^{-1}
$$

The value of entropy of activation $\Delta S_{c}{ }^{0}{ }^{7}$ in standard conditions at $\mathrm{c}_{0}=1 \cdot 10^{6} \mathrm{~mol} \mathrm{~m}^{-3}$ was calculated using the value of pre-exponential factor of the constant $k_{I I}$ andthe Eyring-Wynne-Jones equation and assuming that a transmission factor is equal to unit. It was found that $\Delta S_{c}^{0 \neq}$ is equal to $-190 \mathrm{~J} \cdot \mathrm{mol}^{-1} \mathrm{~K}^{-1}$ and the corresponding steric factor is equal to $1.2 \cdot 10^{-10}$. The latter value is characteristic of bimolecular reactions between polyatomic molecules. The half-life of 1 at $25^{\circ} \mathrm{C}$ calculated using the reaction rate constant is $2.2 \mathrm{~h}$.

\subsection{Measurement of the Solubility of NO in the Water-Dimethyl Sulfoxide Solution}

For the temperature dependence of Henry's law constant $K_{h}$ (Fig. 3) an expression was obtained:

$$
\left.K_{h}=10^{5.9 \pm 0.2} \exp [-(2480 \pm 120) / T)\right] \mathrm{atm} \cdot \mathrm{l} \cdot \mathrm{mol}^{-1}(r=0.980)
$$

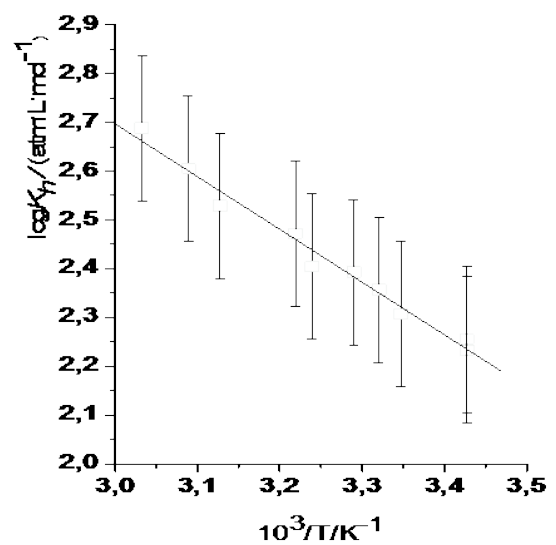

Figure 3. Dependence of Henry's constant for NO dissolved in the mixed $\mathrm{H}_{2} \mathrm{O}$-DMSO solvent versus the reciprocal temperature.

At $25^{\circ} \mathrm{C} K_{h}$ in the mixed solvent is equal to $203 \mathrm{~atm} \cdot 1 \cdot \mathrm{mol}^{-1}$. It is less than that for pure water $530[15]$ and $333[16,17]$ for DMSO, respectively. The same kind of dependence of Henry's constants for the mixed solvent on composition with the extreme in the curve was observed when studying solutions of $\mathrm{N}_{2} \mathrm{O}$ and $\mathrm{CO}_{2}$ in diethanolamine dissolved in water-ethylene glycol composition [18-20].

\subsection{Electrochemical Method}

The total amount of $\mathrm{NO}\left(n_{N O}\right)$ generated during the hydrolytic degradation of the complex and distributed between the solution and the gas phase was calculated when used the measured amounts of $\mathrm{NO}$ in solution $\left(n_{L, N O}\right)$ 
obtained in the electrochemical experiment from the formula:

$$
{ }_{n N O}={ }_{n L, N O}\left(1+K_{h} V_{G} / R T V_{L}\right)
$$

where $V_{G}, V_{L}$ is a volume of the gas phase and solution in the reaction vessel, respectively; $R$ is gas constant, 0.082 $1 \cdot \mathrm{atm} / \mathrm{mol} \cdot \mathrm{K} ; T$ is experimental temperature in Kelvin.

Fig. 4 presents a comparison of the conversion degree of the hydrolytic degradation of $1 \quad \eta$ calculated using microcalorimetric and electrochemical methods. In the latter case $\eta$ is calculated from the formula

$$
\eta=n_{N O} / 4 n_{\text {comp }}
$$

where $n_{\text {comp }}$ is quantity of the complex in the initial solution. It is seen from Fig. 4 that there is a reasonable agreement in the conversion degree measured by two different physicochemical methods.

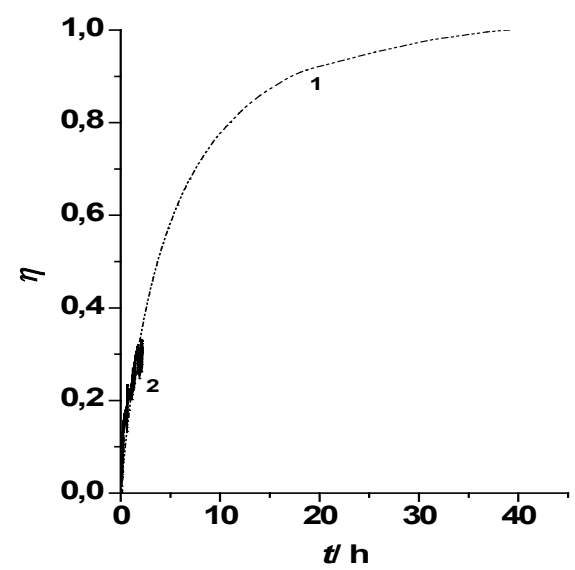

Figure 4. Conversion degree $\eta$ versus time $\mathrm{t} / \mathrm{h}: 1-$ microcalorimetric method, $\mathrm{T}=29.4^{\circ} \mathrm{C} ; 2-$ electrochemical method, $\mathrm{T}=28^{\circ} \mathrm{C}$

\section{Conclusion}

The obtained temperature dependence of reaction rate constant of hydrolytic degradation of 1 allows one to estimate the duration of activity of this potential drug and the possibility of the targeted delivery of NO to cellular targets. It has been shown that the electrochemical method can be used additionally to the microcalorimetric one to estimate hydrolytic stability of the newly synthesized nitrosyl complexes. High enough rates of the hydrolytic degradation of these complexes at high negative entropy of activation can be explained by the low value of activation energy.

\section{Acknowledgements}

The work was supported by the Program of the Presidium of RAS "Fundamental Problems of Medicine".

\section{REFERENCES}

[1] L.J. Ignarro (Editor). Nitric Oxide: Biology and Pathobiology, Academic Press; San Diego, 2000.

[2] R.J. Dai, S.C. Ke. Detection and Determination of the $\{\mathrm{Fe}(\mathrm{NO}) 2\}$ Core Vibrational Features in Dinitrosyl-Iron Complexes from Experiment, Normal Coordinate Analysis, and Density Functional Theory: An Avenue for Probing the Nitric Oxide Oxidation State, J. Phys. Chem. B, Vol.111, 2335-2346, 2007.

[3] A.F. Vanin. Dinitrosyl iron complexes with thiolate ligands: Physico-chemistry, biochemistry and physiology, Nitric Oxide, Vol.21, 1-13, 2009.

[4] L. Li. Some Coordination Chemistry of Non-heme Iron Nitrosyl Complexes, Comm. Inorg. Chem., Vol. 23, 335-353, 2002.

[5] N.A. Sanina, S.M. Aldoshin. Structure and properties of nitrosyl iron complexes with sulfur-containing ligands, Russ. Chem. Bull., Vol.60, 1223-1251, 2011.

[6] N.A. Sanina, T.N. Rudneva, K.A. Lyssenko, O.S. Zhukova, N.S. Emel'yanova, S.M. Aldoshin. Application No. WO 2009/148346 A1.

[7] N.A. Sanina, L.A. Syrtsova, N.I. Shkondina, T.N. Rudneva, A.I. Kotel'nikov, and S.M. Aldoshin. Reaction of ferricytochrome $\mathrm{c}$ with the iron nitrosyl complex $\{\mathrm{Fe} 2[\mathrm{~S}(\mathrm{CH} 2) 2 \mathrm{NH} 3] 2(\mathrm{NO}) 4\} \mathrm{SO} 4 \cdot 2.5 \mathrm{H} 2 \mathrm{O}$, Russ. Chem. Bull, Vol.59, 1565-1571, 2010.

[8] N.A. Sanina, L.A. Syrtsova, B.L. Psikha, N.I. Shkondina, T.N. Rudneva, A.I. Kotel'nikov, S.M. Aldoshin. Ferrocytochrome $\mathrm{c}$ and deoxyhemoglobin in the reaction with the iron cysteaminenitrosyl complex $\{\mathrm{Fe} 2[\mathrm{~S}(\mathrm{CH} 2) 2 \mathrm{NH} 3] 2(\mathrm{NO}) 4\} \mathrm{SO} 4 \cdot 2.5 \mathrm{H} 2 \mathrm{O}$, Russ. Chem. Bull., Vol.59, 1994-1998, 2010.

[9] T.N. Roudneva, N.A. Sanina, K.A.Lyssenko, S.M. Aldoshin, M.Yu. Antipin, N.S.Ovanesyan, Synthesis and structure of water-soluble nitrosyl iron complex with cysteinamine ligand, Mendeleev Communications, Vol.19, 253-255, 2009.

[10] Y.V. Karyakin, I.I. Angelov. Chistye khimicheskie veschestva (Pure chemical substances), Khimia, Moscow, 1974, (in Russian).

[11] A. Weissberger, E.S. Proskauer, J.A. Riddick, E.E. Toops. Organic Solvents. Physical Properties and Methods of Purification, Interscience Publishers Inc., N.Y.-L., 1955.

[12] M. P. Broderick, Z. Taha. Nitric oxide detection using a popular electro-chemical sensor: Recent application and the development of a new generation of highly sensitive and selective NO-microsensors, 4th IBRO World Congress of Neuroscience, Kyoto, Japan; 2-18, 1995.

[13] X. Zhang and M.P. Broderick. Amperometric detection of nitric oxide, Mod. Asp. Immunobiol., Vol.1, 160-165, 2000.

[14] O.S. Gal'yuk, Yu.I. Rubtsov, G.F. Malinovskaya, G.B. Manelis. Microcalorimeter for study kinetics of chemical reactions, Zhurnal Fizicheskoy Khimii, Vol.39, 2319-2322, 1965 (in Russian).

[15] L.H. Gevantman. Solubility of selected gases in water, In: D.R. Lide (Editor). CRC handbook of chemistry and physics. 80th ed., CRC Press, London, 2000. 
[16] P. Ascenzi, M. Brunori, G. Pennesi, C. Ercolani, F. Monacelli. Equilibrium and kinetic study of nitric oxide binding to phthalocyaninatoiron(II) in dimethyl sulphoxide, J. Chem. Soc. Dalton Trans., 369-371, 1987.

[17] R. Ascenzi, R. Fruterro, C. Ercolani, F. Monacelli. Determination of nitric oxide solubility in different solvents via the reaction with phthalocyaninatoiron(II), Analusis, Vol. 24, 316-318, 1996.

[18] G. F.Versteeg, W.P.M. Swaal. Solubility and diffusivity of acid gases (carbon dioxide, nitrous oxide) in aqueous alkanolamine solutions, J. Chem. Eng. Data, Vol.33, 29-34, 1988.
[19] M.H. Oyevaar, R.W.J. Morssinkhof, K.R. Westerterp. Density, viscosity, solubility, and diffusivity of carbon dioxide and nitrous oxide in solutions of diethanolamine in aqueous ethylene glycol at $298 \mathrm{~K}$, J. Chem. Eng. Data, Vol.34, 77-82, 1989.

[20] M.H. Oyevaar, H.J. Fontein, K.R. Westerterp. Equilibria of carbon dioxide in solutions of diethanolamine in aqueous ethylene glycol at 298 K, J. Chem. Eng. Data, Vol.34, 405-408, 1989. 